\title{
Health monitoring of Ceramic Matrix Composites from waveform-based analysis of Acoustic Emission
}

\author{
Emmanuel Maillet ${ }^{\mathrm{a}}$, Matthew P. Appleby and Gregory N. Morscher \\ The University of Akron, Mechanical Engineering Department, Akron, OH 44325-3903, USA
}

\begin{abstract}
Ceramic Matrix Composites (CMCs) are anticipated for use in the hot section of aircraft engines. Their implementation requires the understanding of the various damage modes that are involved and their relation to life expectancy. Acoustic Emission (AE) has been shown to be an efficient technique for monitoring damage evolution in CMCs. However, only a waveform-based analysis of $\mathrm{AE}$ can offer the possibility to validate and precisely examine the recorded $\mathrm{AE}$ data with a view to damage localization and identification. The present work fully integrates wave initiation, propagation and acquisition in the analysis of Acoustic Emission waveforms recorded at various sensors, therefore providing more reliable information to assess the relation between Acoustic Emission and damage modes. The procedure allows selecting AE events originating from damage, accurate determination of their location as well as the characterization of effects of propagation on the recorded waveforms. This approach was developed using AE data recorded during tensile tests on carbon/carbon composites. It was then applied to meltinfiltrated $\mathrm{SiC} / \mathrm{SiC}$ composites.
\end{abstract}

\section{Introduction}

Ceramic matrix composites (CMCs) are being considered for aerospace applications including combustor liners and rotating components in the next generation jet engines. Their successful implementation requires the understanding of the various damage modes that are involved and their impact on life expectancy. Among the numerous existing health monitoring techniques, Acoustic Emission (AE) is unique in that it is based on the recording and analysis of elastic waves that are generated by material damage. Using few remote sensors, AE allows real-time monitoring and localization of damage. In CMCs, the cumulative energy of acoustic emission signals has been shown to be directly related to transverse matrix crack density [1, 2]. In static fatigue at intermediate temperatures, the energy of $\mathrm{AE}$ events has also been used as an indicator for lifetime prediction [3, 4]. Finally, one of the main current challenges is the use of AE for damage identification, based on the assumption that AE signals carry specific features related to their source damage. Various attempts have been made on polymer and ceramic matrix composites [5-8].

The literature presents promising results showing the ability of Acoustic Emission to monitor and identify damage in composites. However, results can hardly be compared and the developed approaches

\footnotetext{
${ }^{\text {a }}$ Corresponding author: emaillet@uakron.edu
}

This is an Open Access article distributed under the terms of the Creative Commons Attribution License 4.0, which permits unrestricted use, distribution, and reproduction in any medium, provided the original work is properly cited. 


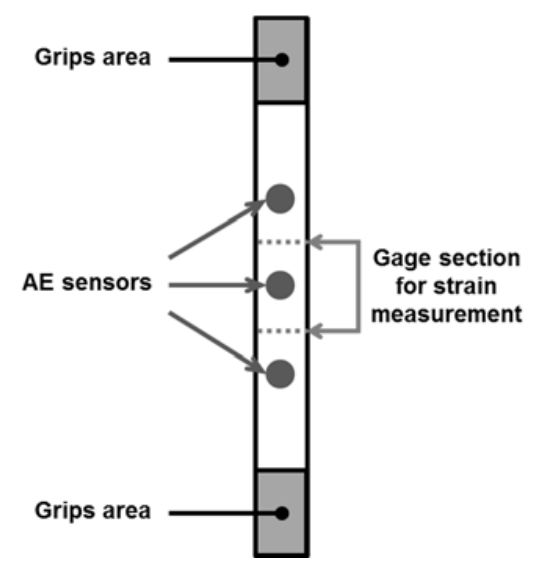

Figure 1. Typical 3-sensor configuration for AE monitoring during tensile testing of a composite specimen. In this configuration, AE events triggering the middle sensor first originate from the gage section.

cannot easily be transposed to other composite systems because most works (1) suffer from the absence of a robust procedure for the selection and analysis of AE signals originating from damage and (2) fail to account for effects of propagation on the recorded AE waveforms. This paper will discuss both aspects using a waveform-based procedure that includes accurate location of $\mathrm{AE}$ events and comparison of signal energy at two sensors. The procedure allows selection of AE events originating from damage [9] as well as characterization of effects of propagation on the recorded waveforms. This type of analysis is made possible by the use of Modal Acoustic Emission (MAE). MAE has two main advantages compared to traditional AE. First, MAE uses wide-band transducers with a high sensitivity in the range $50 \mathrm{kHz}-$ $2 \mathrm{MHz}$ against resonant transducers sensitive around $500 \mathrm{kHz}$ in traditional AE. Second, the analysis is based on the recorded waveforms and not only on discrete signal features.

Two composite systems were studied. The first composite system, a carbon fiber reinforced carbonsilicon carbide matrix composite $\left(\mathrm{C}_{\mathrm{f}} / \mathrm{C}\right.$-SiC), serves as a model material since only few $\mathrm{AE}$ events are generated by damage and no significant changes in the wave propagation properties of the material are expected due to damage progression. The second composite system is a state-of-the-art SiC fiber reinforced, melt-infiltrated $\mathrm{SiC}$ matrix composite $(\mathrm{SiC} / \mathrm{SiC})$. In this case, thousands of $\mathrm{AE}$ events are generated by damage, which are expected to be related to various damage modes (matrix cracking, interfacial phenomena, fiber failures). Moreover, the matrix experiences significant damage under tensile loading and therefore substantial changes in the wave propagation properties of the material are expected.

\section{Materials and $A E$ data acquisition}

The AE data used in this paper were recorded during tensile tests at room temperature on two types of ceramic matrix composites: woven carbon fiber reinforced carbon-SiC matrix composites $\left(\mathrm{C}_{\mathrm{f}} / \mathrm{C}-\mathrm{SiC}\right)$, with a resin-derived carbon matrix and a chemically vapor infiltrated $\mathrm{SiC}$; and woven $\mathrm{Hi}$ Nicalon $\mathrm{SiC}$ fiber reinforced melt-infiltrated $\mathrm{SiC}$ matrix composites $(\mathrm{SiC} / \mathrm{SiC})$. The $\mathrm{C}_{\mathrm{f}} / \mathrm{C}-\mathrm{SiC}$ specimens were straight-sided coupons of $152 \mathrm{~mm}$ in length, $12.7 \mathrm{~mm}$ in width and $1.2 \mathrm{~mm}$ in thickness. The $\mathrm{SiC} / \mathrm{SiC}$ specimens were machined into dogbones; the width of the gage was $8.3 \mathrm{~mm}$ and the thickness nominally $3.4 \mathrm{~mm}$. Three AE sensors (ref. B1025, Digital Wave Corporation) were installed in the configuration presented in Fig. 1 and connected to a Fracture Wave Detector acquisition system 
(a)
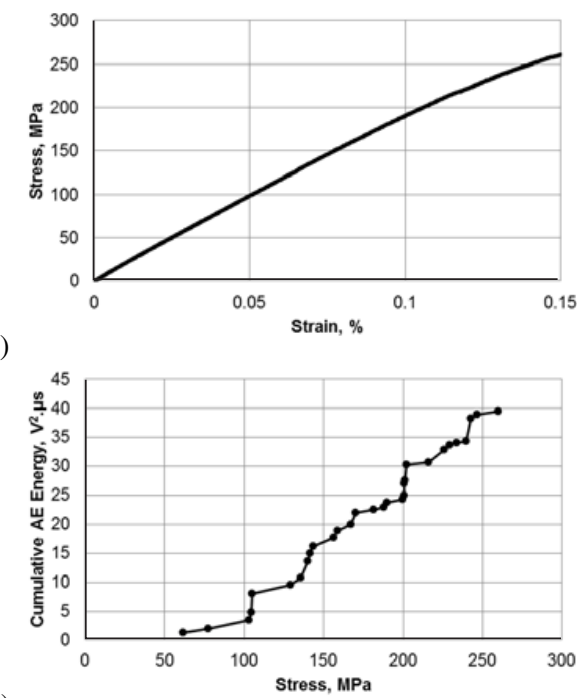

(b)

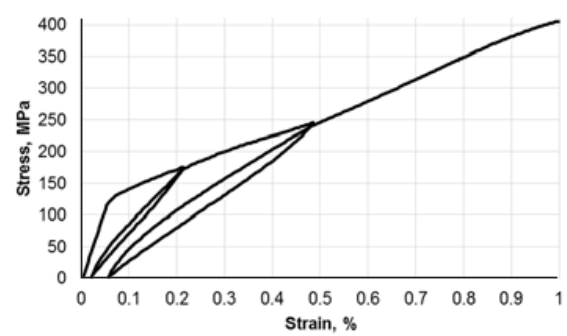

(c)

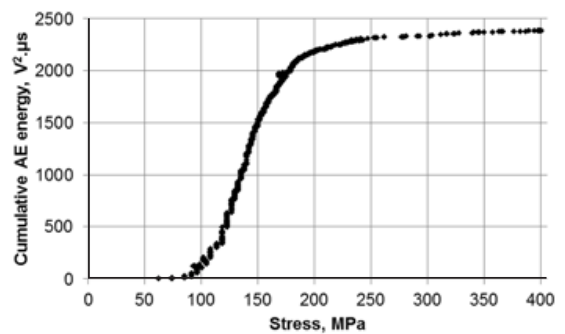

(d)

Figure 2. Characteristic stress-strain and cumulative $\mathrm{AE}$ energy vs. stress curves for a $\mathrm{C}_{\mathrm{f}} / \mathrm{C}-\mathrm{SiC}$ composite (a-b) and a $\mathrm{SiC} / \mathrm{SiC}$ composite (c-d).

(Digital Wave Corporation, Centennial, CO). Each AE signal consisted of 1024 points digitized at a rate of $10 \mathrm{MHz}$, giving a recorded duration of $102.4 \mu \mathrm{s}$. The first $25 \%$ of each signal, called the pretrigger, corresponded to points recorded before the system triggered. The three sensors were enslaved to one another, meaning that when any of the sensors was triggered all three sensors recorded a signal simultaneously, therefore ensuring that the three AE signals corresponded to the same event. In the present configuration, the events occurring in the gage section trigger the middle sensor first. Characteristic stress vs. strain and cumulative AE energy vs. stress curves are shown in Fig. 2 for both composite systems. 


\section{MATEC Web of Conferences}

$\mathrm{AE}$ data corresponding to artificial $\mathrm{AE}$ sources generated on $\mathrm{C}_{\mathrm{f}} / \mathrm{C}-\mathrm{SiC}$ specimens prior to testing were also analyzed in order to validate the selection procedure. Two AE sensors were placed $60 \mathrm{~mm}$ away in a configuration similar to the top and bottom sensors used for tensile testing (Fig. 1). The artificial sources were generated at various locations along the specimen's length either by breaking $0.5 \mathrm{~mm}$ diameter pencil leads (Hsu-Nielsen sources) or $0.2 \mathrm{~mm}$ diameter boron fibers (Specialty Materials, Inc., Lowell, MA).

Finally, ultrasonic measurements were also performed on $\mathrm{C}_{\mathrm{f}} / \mathrm{C}-\mathrm{SiC}$ specimens prior to tensile testing in order to characterize energy attenuation. Two AE sensors were placed $60 \mathrm{~mm}$ away in a configuration similar to the top and bottom sensors of Fig. 1. A third AE sensor of the same type (ref. B1025) placed outside of the first two was used to generate single-frequency waveforms at 300, 600, 900, 1200 and $1500 \mathrm{kHz}$ with constant amplitude. Energy attenuation was calculated based on signal energy recorded at the two sensors in the first $50 \mu$ s after signal onset.

\section{Waveform-based analysis of AE data}

\subsection{Limits of the existing selection procedure}

During mechanical testing of a typical $150 \mathrm{~mm}$ long composite dogbone specimen 70 to $80 \%$ of the recorded $\mathrm{AE}$ originates from outside the gage section (i.e. for a tensile test, typically a $25.4 \mathrm{~mm}$ long zone in the center of the testing specimen where strain is measured) [10]. If not properly filtered out, these AE signals can greatly affect the analysis. Therefore, when attempting to relate acoustic emission to a material's mechanical behavior, the analysis must be limited to AE events generated by the material within the gage section. These AE events are here referred to as gage events. In the 3 -sensor configuration (Fig. 1), the selection of gage events is straightforward as those events trigger the middle sensor first. Events triggering one of the guard sensors (top or bottom sensor) first might be associated to damage occurring between the top and bottom sensors, but could also originate from noiserelated signals generated in the grips. The 3-sensor configuration cannot always be applied because of testing conditions (e.g. test at high temperature) or sample geometry (e.g. single-tow minicomposites). Therefore, the selection procedure must ensure that, in tests where only two sensors are used, the analyzed AE events were indeed generated by damage occurring within the gage section.

The location of AE events is commonly employed as a selection parameter when only two AE sensors are used (one placed on each side of the gage section). From the times of arrival at top and bottom sensors, $t_{\text {top }}$ and $t_{\text {bottom }}$ respectively, the location $l$ of an event along the specimen's longitudinal axis is $l=V\left(t_{\text {bottom }}-t_{\text {top }}\right) / 2$, where $V$ is the wave velocity. In composite materials, wave velocity varies with frequency. The recorded AE signals correspond mostly to the zero-order flexural and extensional modes, $A_{0}$ and $S_{0}$ respectively. In the frequency range considered in acoustic emission (up to $2 \mathrm{MHz}$ ), the velocity of the high-frequency $S_{0}$ mode remains constant while that of the lower frequency $A_{0}$ mode can vary greatly. Therefore, accurate location of AE events relies on the detection of the highfrequency extensional mode $S_{0}$, which requires the use of wide-band sensors [11]. The determination of time of arrival is critical in order to accurately locate AE events. Signal onset picking can be performed manually when the number of AE events is limited. However, manual signal onset determination is usually not employed in the case of AE monitoring of composites since processing the thousands of events that are recorded would be extremely time consuming. The most widely used technique for automatic onset detection - usually performed in real-time on acquisition systems - is the firstthreshold-crossing technique. The signal onset is considered as the first point in the signal exceeding a fixed threshold value. However, the use of a fixed threshold value can cause late triggering, missing low amplitude extensional waves, or undesired triggering on noise. To overcome the limitations of the first-threshold-crossing technique, a technique based on the Akaike Information Criterion (AIC) was recently developed by Sedlak et al. for AE signal onset picking [12]. In this paper, manual 
determination and the so-called first-threshold-crossing technique are compared to the AIC-based technique.

\subsection{Accurate location of AE events}

The AIC-based technique [12] consists in calculating for each point $k$ of a signal $x$ composed of $N$ points, the similarity between the signal portion prior to and after the considered point $k$ :

$$
A I C(k)=k \log _{10}[\operatorname{var}(x[1: k])] \ldots+(N-k-1) \log _{10}[\operatorname{var}(x[k+1: N])]
$$

The resulting function, called AIC function, reaches a minimum value at the waveform onset when the similarity is a minimum between noise prior to onset and highly-correlated signal after the onset.

The technique developed by Sedlak et al. showed significant improvement when compared to the first-threshold-crossing technique for AE signals recorded during a tensile test on a thin metal plate [12]. The technique is briefly summarized here. Further details are available in [12]. To calculate the AIC function, the two-step procedure uses a characteristic function, which is calculated from the $\mathrm{AE}$ waveform in order to enhance amplitude and frequency changes within the signal and thus improve onset determination. The characteristic function is expressed as follows:

$$
C F(k)=|x(k)|+R|x(k)-x(k-1)|
$$

where $x$ is the AE signal and $R$ is a constant determined empirically.

The procedure also involves three empirical time constants $\Delta t_{A M}, \Delta t_{F B}$ and $\Delta t_{F A}$, which are used to narrow the time window of interest for signal onset determination. The first step consists in calculating the AIC function and finding signal onset using a truncated signal that starts at $t=0$ (beginning of pretrigger) and ends $\Delta t_{A M}$ after the peak of highest magnitude. In the second step, the AIC function is computed again, this time truncating the signal between $\Delta t_{F B}$ before the onset found in the first step and $\Delta t_{F A}$ after that onset. The signal onset is then refined.

For each composite system considered in this paper, optimum values of the four aforementioned parameters were determined by comparing signal onsets obtained for various sets of values with those obtained by manual determination. Comparison with the first-threshold-crossing technique will be developed for the $\mathrm{SiC} / \mathrm{SiC}$ composite system only, since the number of $\mathrm{AE}$ events recorded in $\mathrm{C}_{\mathrm{f}} / \mathrm{C}$ $\mathrm{SiC}$ composites was very limited.

\subsection{Selection of AE events based on recorded energies}

An energy-based method is used here to validate the selection of AE events based on location. Indeed, location alone does not ensure that an $\mathrm{AE}$ event was generated in the gage section. Two separate noise signals could trigger top and bottom sensors with a difference of arrival times similar to that of a gage event and therefore be considered as such. The proposed method compares the amount of energy recorded by top and bottom sensors and can assess that both recorded AE signals were generated by the same gage event. This method was recently developed in order to monitor damage progression in ceramic matrix composites under static loading by in-situ determination of energy attenuation [4]. The approach could not be demonstrated, however, since the AE waveforms were not recorded and the analysis only relied on discrete signal features. Therefore, in the present paper, the energy-based approach is first demonstrated and then applied for the selection of AE events originating from damage.

The following expression has been proposed for the energy recorded by sensor $i$ for an AE event $n[4]:$

$$
E_{i}(n)=E_{S}(n) A_{i} e^{-B d_{i}(n)}
$$




\section{MATEC Web of Conferences}

$E_{s}(n)$ is the energy released at the source $n$ in the forms of elastic waves. In practice, the recorded energy $E_{i}$ ranges over several orders of magnitude, which is attributed mostly to the scatter on released energy $E_{s}$. The recorded energy $E_{i}(n)$ decreases exponentially with propagation distance $d_{i}(n)$ from the source to sensor $i, B$ being the attenuation coefficient. The amount of recorded energy is also affected by the sensor's frequency response and coupling to the specimen's surface, which are characterized by $A_{i}$, a characteristic of sensor $i$ ranging from 0 to 1 and considered constant for a given test. It is worth noting that, even though the frequency response of a sensor can be accurately determined, coupling will vary from one test to another. Therefore, $A_{i}$ is unknown. It is also important to note that composite materials exhibit frequency-dependent attenuation [13-15]. Therefore, $B$ is expected to vary depending on the frequency content of the generated signal and so is $A_{i}$ since it describes the sensor's frequency response.

The energy ratio between the top and bottom sensors can then be defined as:

$$
R(n)=\operatorname{In}\left(E_{\text {top }}(n) / E_{\text {bottom }}(n)\right)
$$

where $E_{\text {top }}$ and $E_{\text {bottom }}$ are the recorded energies at top and bottom sensors, respectively. Using Eq. (3) and replacing $d_{i}(n)$ by $d_{\text {top }}(n)=L-l(n)$ or $d_{\text {bottom }}(n)=L+l(n)$ (where $L$ is the distance between sensor and specimen center and $l$ is the location along the longitudinal axis with $l=0$ at the center), the energy ratio can be expressed as:

$$
R(n)=2 B l(n)+\operatorname{In}\left(A_{\text {top }} / A_{\text {bottom }}\right)
$$

The energy ratio is a linear function of event location $l$, of slope $2 B$ and ordinate at the origin $\ln \left(A_{\text {top }} / A_{\text {bottom }}\right)$. In practice, for all AE events recorded during one test, one can represent their energy ratio as a function of location. The overall trend allows identifying the attenuation coefficient $B$ from the slope, and the ratio $A_{\text {top }} / A_{\text {bottom }}$ from the ordinate at the origin. Even though $A_{\text {top }}$ and $A_{\text {bottom }}$ are unknown, the ratio $A_{\text {top }} / A_{\text {bottom }}$ indicates which sensor recorded the most energy for events located midway between the two and therefore indicates which sensor was the most sensitive. The most sensitive sensor is the top sensor if $\ln \left(A_{\text {top }} / A_{\text {bottom }}\right)>0$ and the bottom sensor otherwise. Finally, a slight scatter is expected around the linear trend due to uncertainties in location and energy ratio, which are discussed in the Results section. However, if an event is far from the overall linear trend, it is most likely to have not originated from damage.

\subsection{Characterization of effects of propagation}

Energy attenuation of elastic waves in composite materials depends strongly on wave frequency [13-15], higher frequency waves experiencing greater attenuation. The characterization of frequency-dependent attenuation is therefore necessary when attempting to relate AE signal features to damage mechanisms. The energy-based approach described in the previous section can be used to characterize frequencydependent attenuation by considering the signal energy contained in various consecutive frequency intervals instead of the total signal energy.

Based on Parseval's theorem, the total energy $E_{t}$ contained in a waveform $x(t)$ is equal to the total energy of the waveform's Fourier transform $X(f)$, which can be written as follows for the discrete Fourier transform $X$ of a signal $x$, both of length $N$ :

$$
E_{t}=\sum_{n=0}^{N-1} x(n)^{2}=\frac{1}{N} \sum_{k=0}^{N-1} X(k)^{2}
$$

In the Digital Wave system used in the present work, the signal energy defined in $\mathrm{V}^{2} . \mu$ s corresponds to $E_{t} / f_{s}$, where $f_{s}$ is the sampling frequency. Therefore, the energy calculated in the frequency domain can be divided by $f_{s}$ as well for consistency. The partial energy $E_{p}\left(f_{i} ; f_{j}\right)$ contained in the frequency interval $\left[f_{i} ; f_{j}\right]$ (or the interval $\left[k_{i} ; k_{j}\right]$ if expressed in terms of elements of the discrete Fourier 
transform) is given by the following equation, in which the factor 2 in the numerator takes into account the symmetry of the Fourier transform:

$$
E_{p}\left(f_{i} ; f_{j}\right)=\frac{2}{f_{s} N} \sum_{k=k_{i}}^{k j} X(k)^{2}
$$

By using this partial energy to calculate the energy ratio, it is possible to identify the attenuation coefficient associated with a specific frequency band. In practice, for the same AE events generated during a mechanical test, various energy ratio vs. location plots can be obtained for different frequency ranges defined within the sensitivity range of the $\mathrm{AE}$ sensors $(50 \mathrm{kHz}$ to $2 \mathrm{MHz})$. The attenuation coefficient associated with each frequency range can then be extracted from the slope of the linear fit. This approach offers the possibility of using only AE events recorded during mechanical tests to characterize frequency-dependent attenuation and does not require additional ultrasonic measurements.

\section{Application of the waveform-based analysis to $\mathrm{C}_{\mathrm{f}} / \mathrm{C}$-SiC composites}

\subsection{Demonstration of the energy-based approach}

In order to demonstrate the validity of the energy-based approach, artificial AE sources were generated on the surface of $\mathrm{C}_{\mathrm{f}} / \mathrm{C}-\mathrm{SiC}$ tensile coupons as described in Sect. 2. For each artificial AE event, the location was calculated based on manually-determined signal onsets in order to minimize location uncertainties and therefore focus on the validation of the energy-based approach. The energy ratio was calculated and reported on the energy ratio vs. location plot. Results are shown in Fig. 3(a-b) for both pencil lead breaks and boron fiber breaks.

In both cases, experimental results are in excellent agreement with the expected linear trend, which demonstrates the validity of the proposed model for recorded AE energy. The attenuation coefficient, identified from the slope of the linear fit, is $2.5 \times 10^{-2} \mathrm{~mm}^{-1}$ for pencil leads and $3 \times 10^{-2} \mathrm{~mm}^{-1}$ for boron fibers. The limited scatter is mainly attributed to location uncertainties and variations in source frequency. Although location was calculated based on manually-determined signal onsets, uncertainties remain due to location resolution. Signals were sampled at a rate of $10 \mathrm{MHz}$, therefore the time uncertainty at onset picking was $0.1 \mu \mathrm{s}$. Considering a wave velocity of $10,000 \mathrm{~m} / \mathrm{s}$, which is common in CMCs, the time uncertainty leads to a location resolution of $\pm 1 \mathrm{~mm}$. Scatter in energy ratio can be caused between two AE events by differences in frequency content leading to differences in attenuation coefficient. The frequency content of generated AE sources was described by the frequency centroid (weighted frequency average) of signals recorded after limited propagation throughout the material ( 0 to $20 \mathrm{~mm}$ ). The frequency centroid ranged from 200 to $350 \mathrm{kHz}$ for pencil lead breaks and from 700 to $800 \mathrm{kHz}$ for boron fiber breaks. It appears that for similar amplitude of frequency centroid range, the scatter in energy ratio is similar. Moreover, as expected, lower source frequency leads to lower attenuation.

\subsection{Selection of AE events generated by damage}

All the specimens tested exhibited similar AE behavior. About 30 gage events (that triggered the middle sensor first) were recorded and the AE energy release was significant beyond $100 \mathrm{MPa}$ (Fig. 2b). Since the non-linearity on the stress-strain curve (generally associated with matrix cracking and fiber sliding) was very limited (Fig. 2a), these events were associated to fiber failures. The energy ratio vs. location plot was generated for each test and very consistent results were observed. Note that for each $\mathrm{AE}$ event, location was calculated based on manually-determined signal onsets in order to minimize location uncertainty. Results are shown for two tests in Fig. 3(c-d). Similar to artificial AE events, damage-related $\mathrm{AE}$ events were in very good agreement with the expected linear trend. The attenuation coefficient, identified from the slope of the linear fit, was $7 \times 10^{-2} \pm 1 \times 10^{-2} \mathrm{~mm}^{-1}$ (mean \pm standard 


\section{MATEC Web of Conferences}

(a)
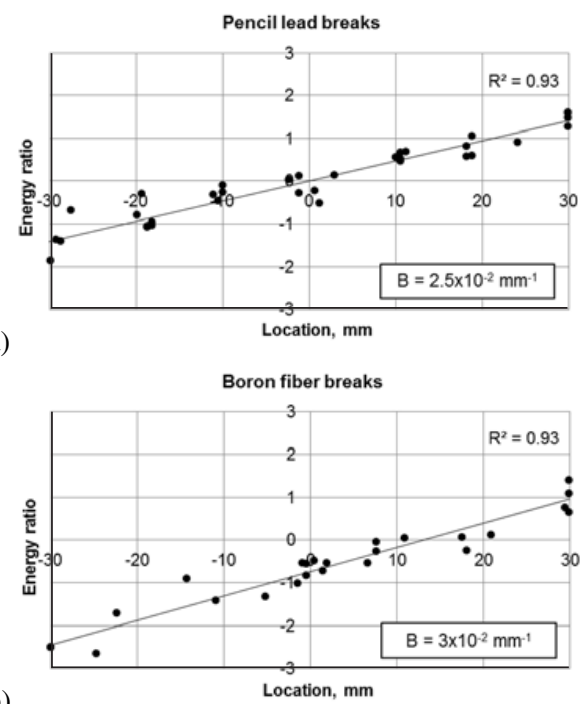

(b)

Location, $\mathrm{mm}$

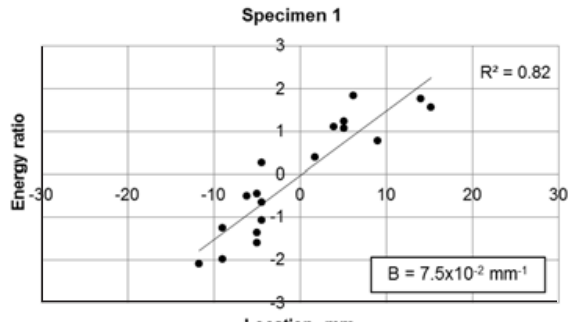

(c)

Location, $\mathrm{mm}$

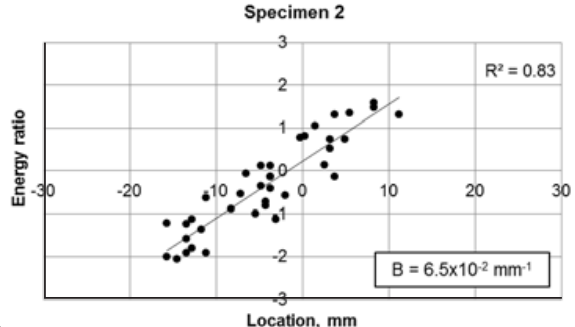

(d)

Figure 3. Energy ratio vs. event location along the longitudinal axis for $\mathrm{C}_{\mathrm{f}} / \mathrm{C}-\mathrm{SiC}$ composites with artificial sources (a-b) and AE events generated by damage during two room temperature tensile tests (c-d). Note: location was determined by manual selection of AE signal onsets.

deviation). The greater attenuation coefficient value and scatter observed on the energy ratio vs. location plot when compared to results obtained with pencil lead or boron fiber breaks are both attributed to differences in source frequency content. For the damage-related events, the frequency centroid of signals recorded after limited propagation throughout the material (0 to $20 \mathrm{~mm}$ ) ranged from 800 to $1200 \mathrm{kHz}$. As would be expected, higher frequency waves experience higher attenuation. Moreover, the greater scatter in energy ratio is attributed to the greater range in source frequency ( $400 \mathrm{kHz}$ for damage events against $100-150 \mathrm{kHz}$ for artificial events).

Finally, to illustrate the use of the energy ratio vs. location plot as a visual tool to assess the selection of $\mathrm{AE}$ events originating from damage, all the $\mathrm{AE}$ events recorded during test were considered and 


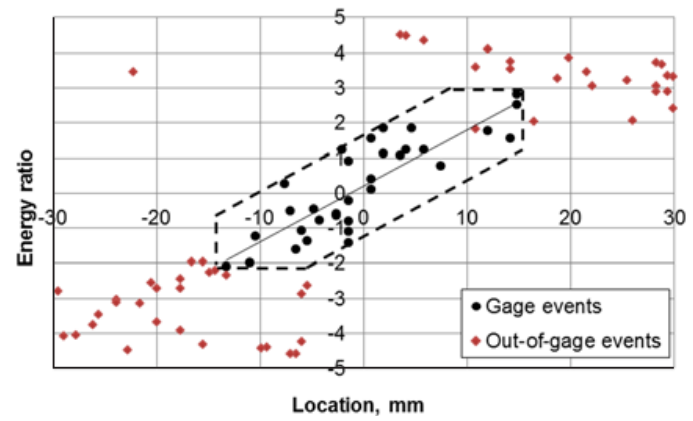

Figure 4. Energy ratio vs. event location (determined automatically using AIC) for all events recorded during room temperature tensile test of a $\mathrm{C}_{\mathrm{f}} / \mathrm{C}-\mathrm{SiC}$ composite.

the energy ratio vs. location plot was generated automatically using the AIC technique for location determination (Fig. 4). Optimum location accuracy was achieved with the following parameters: $\mathrm{R}=0, \Delta t_{A M}=20 \mu \mathrm{s}, \Delta t_{F B}=30 \mu \mathrm{s}$ and $\Delta t_{F A}=30 \mu \mathrm{s}$. Note that only AE signals recorded by the top and bottom sensors are needed to generate this plot. The true gage events are highlighted here based on the triggering of the middle sensor. Only those events clearly follow the expected linear trend of energy ratio vs. location. Therefore, they could accurately be selected based on this plot in tests where the use of a middle sensor is not possible. Events for which the automatic location determination would attribute a location within the gage section even though they did not trigger the middle sensor first can easily be filtered out based on their energy ratio value (these events are outside the selection box in Fig. 4).

\subsection{Characterization of effects of propagation}

Effects of propagation on recorded AE waveforms can be illustrated by looking at the three waveforms recorded for a same event (Fig. 5). Event location is determined from the difference in arrival times between top and bottom sensors. Therefore, the propagation distance from the source to each sensor is known. In the present example, the middle sensor is the closest to the source $(6 \mathrm{~mm})$. The bottom sensor is $28 \mathrm{~mm}$ away from the source and the top sensor $37 \mathrm{~mm}$ away. In the time domain (Fig. 5a), effects of attenuation appear clearly, the waveforms exhibiting a significant decrease in wave amplitude with increasing propagation distance. Effects of dispersion mentioned in Sect. 3.1 are also visible, especially at the sensor furthest from the source (top sensor here). The high-frequency extensional wave $\left(S_{0}\right)$ propagating at higher velocity reached the sensor first, at about $25 \mu \mathrm{s}$, whereas the lower frequency flexural wave $\left(A_{0}\right)$ travelling at significantly lower velocity is only visible beyond $40 \mu \mathrm{s}$. In the frequency domain, all three spectra show three main peaks at similar frequencies: $300 \mathrm{kHz}, 900 \mathrm{kHz}$ and $1500 \mathrm{kHz}$. However, the distribution of energy between those three peaks is significantly different. The frequency spectrum at the middle sensor, closest to the source, is dominated by the highest frequency peak. At the bottom sensor, all three peaks have similar amplitudes. Finally, at the top sensor, furthest from the source, the frequency spectrum is dominated by the lowest frequency peak. This illustrates the strong frequency dependence of energy attenuation in this composite system. While the energy carried by the lowest frequency component remains almost constant, the energy carried by the highest frequency component is attenuated greatly over the limited observed distance $(31 \mathrm{~mm}$ from middle to top sensor). Therefore, when using remote sensors and attempting to relate AE signal features to damage mechanisms, frequency-dependent attenuation should be characterized and accounted for.

The energy-based approach presented here allows for the characterization of frequency-dependent attenuation. Following the method described in Sect. 3.4, attenuation coefficients were determined for AE events generated by artificial sources as well as by damage during tensile test (Fig. 6). For each 

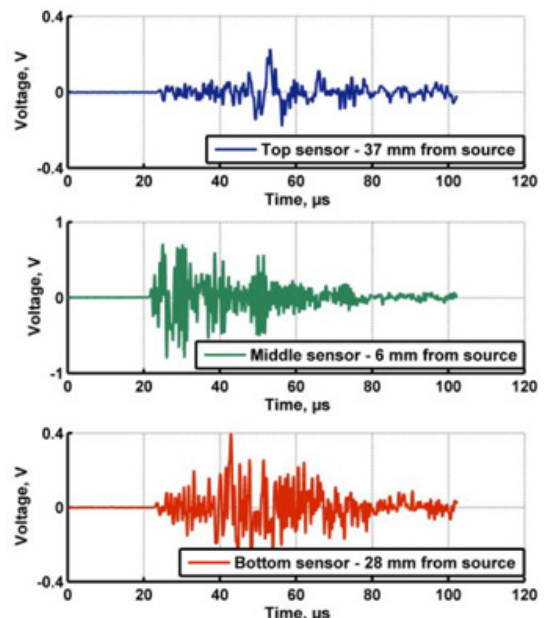

(a)
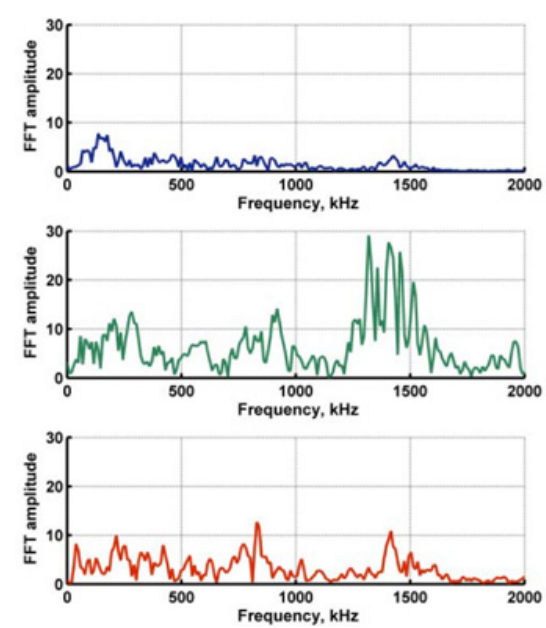

(b)

Figure 5. Recorded waveforms (a) and corresponding Fourier transforms (b) of an AE event generated $6 \mathrm{~mm}$ below the middle sensor at an applied stress of $143 \mathrm{MPa}$ during tensile test of a $\mathrm{C}_{\mathrm{f}} / \mathrm{C}-\mathrm{SiC}$ composite.

set of events, three consecutive frequency intervals centered on the three main peaks identified in Fig. 5 were considered: 50 to $600 \mathrm{kHz}, 600$ to $1200 \mathrm{kHz}$ and 1200 to $1800 \mathrm{kHz}$. Values of attenuation coefficients were extracted from linear fits of the corresponding energy ratio vs. location plots. The error bars in Fig. 6 were calculated considering a 95\% confidence interval around the linear fits. Both sets of results exhibit a very similar trend, showing a significant increase in attenuation at higher frequencies, which for the damage-related events is in excellent agreement with results obtained by ultrasonic measurements following the procedure described in Sect. 2. The underestimate of attenuation coefficient values obtained with artificial sources is attributed to slight signal saturation observed on waveforms recorded at the sensor closest to the source, which led to an underestimate of recorded energy. Finally, the deviation between ultrasonic measurements and in-situ characterization at low frequencies is attributed to a greater sensitivity of energy ratio to the considered signal duration at those frequencies. 


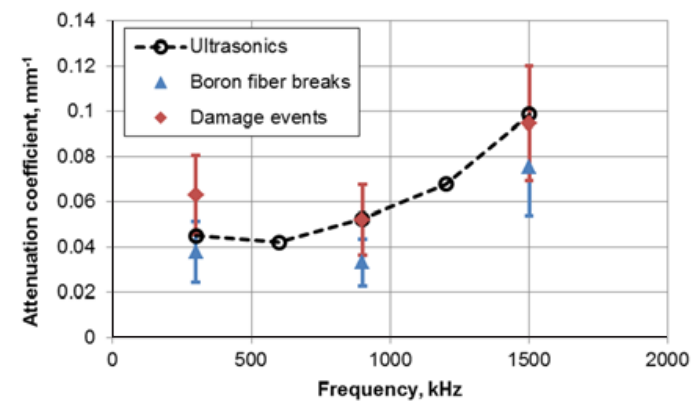

Figure 6. Attenuation coefficient vs. frequency determined by ultrasonic measurements and using the energy-based approach with artificial or damage-related AE events.

In conclusion, these preliminary results show the ability of the energy-based approach to characterize frequency-dependent attenuation in ceramic matrix composites using only acoustic emission data recorded during mechanical tests.

\section{Application of the waveform-based analysis to SiC/SiC composites}

\subsection{Location of AE events}

The tested $\mathrm{SiC} / \mathrm{SiC}$ specimens exhibited comparable AE behavior. A total of about $4000 \mathrm{AE}$ events were recorded throughout each tensile test, and around $25 \%$ of the total were gage events (triggered the middle sensor first). The three AE signals of each gage event were manually inspected for validation and determination of arrival times at top and bottom sensors. This section will focus on the results obtained for two tests with different sensor/material coupling conditions. In Test 1, top and bottom sensors are assumed to have similar coupling conditions with the specimen's surface. Coupling quality is characterized by the average energy recorded by each sensor for all the gage events. In Test 1 , the average recorded energy was $1.6 \mathrm{~V}^{2} . \mu \mathrm{s}$ for the top sensor and $2.9 \mathrm{~V}^{2}$. $\mu \mathrm{s}$ for the bottom sensor. In Test 2, top and bottom sensors are assumed to have very different coupling conditions, and the bottom sensor in particular seemed to have degraded coupling quality. Indeed, the average recorded energy in Test 2 was $5 \mathrm{~V}^{2}$. $\mu$ s for the top sensor and $0.7 \mathrm{~V}^{2}$. $\mu$ s for the bottom sensor. It is worth noting that variations in coupling quality are common in Acoustic Emission mainly because of variations in specimen surface conditions and amount of coupling agent used. Moreover, it is difficult in practice to accurately characterize coupling quality before test therefore leading to the wide range of recorded energies observed here.

One characteristic feature of $\mathrm{SiC} / \mathrm{SiC}$ composites is a strong damage dependence of wave velocity [16] that needs to be accounted for in order to ensure accurate location of AE events throughout tests. Wave velocity is proportional to $\sqrt{E / \rho}$, where $E$ is the material's elastic modulus and $\rho$ its density. Transverse matrix cracking causes a decrease in elastic modulus and thus a decrease in wave velocity. Wave velocity can be monitored throughout testing using the recorded AE signals that triggered top or bottom sensor first and exhibited a clear separation between extensional and flexural modes at the furthest sensor, thus indicating that the wave had propagated over a long distance and that the event originated from outside the sensors. Wave velocity was calculated from the known distance between top and bottom sensors and the manually-determined difference in arrival times. Results are presented in Fig. 7 for Test 2. Wave velocity decreases significantly during test, from over $10,000 \mathrm{~m} / \mathrm{s}$ initially to about $6,000 \mathrm{~m} / \mathrm{s}$ near failure (Fig. 7a). The comparison with elastic modulus (taken as the tangent modulus at beginning of unload for each unload/reload cycle) in Fig. 7b confirms the direct relationship 


\section{MATEC Web of Conferences}

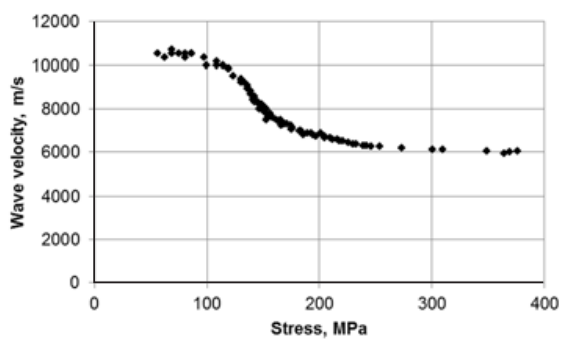

(a)

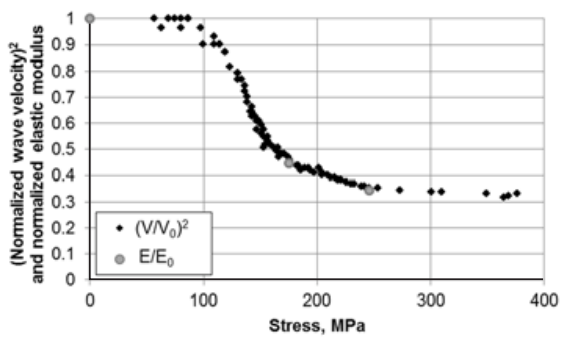

(b)

Figure 7. Damage dependence of wave velocity in a $\mathrm{SiC} / \mathrm{SiC}$ composite. (a) Wave velocity determined from $\mathrm{AE}$ events that occurred outside the outermost sensors vs. applied stress. (b) Comparison with elastic modulus (tangent modulus at beginning of unload) extracted from unload/reload cycles.

between wave velocity and transverse matrix cracking. For the determination of location of gage events, wave velocity was determined from Fig. 7 based on the stress at which the event was recorded.

The performance of both signal onset determination techniques was first evaluated in terms of location accuracy. For both tests, manually-determined arrival times at top and bottom sensors for all gage events served as reference. Event location accuracy relies on the accuracy in difference of arrival times between top and bottom sensors rather than accuracy in arrival time itself. Therefore, the absolute deviation in difference of arrival times was calculated for each AE event between manual determination and first-threshold or AIC techniques. The absolute deviation in location was estimated from the deviation in difference of arrival times assuming a constant wave velocity of $10,000 \mathrm{~m} / \mathrm{s}$ for simplicity. The parameters leading to optimum results were $20 \mathrm{mV}$ for the first-threshold technique and $\mathrm{R}=1, \Delta t_{A M}=20 \mu \mathrm{s}, \Delta t_{F B}=30 \mu \mathrm{s}$ and $\Delta t_{F A}=10 \mu \mathrm{s}$ for the AIC technique. On average, the AIC technique slightly outperforms the first-threshold-crossing technique leading to $5 \%$ more events located with a deviation of $\pm 4 \mathrm{~mm}$ from manual determination. Moreover, coupling quality seems to have a significant effect on location accuracy for both techniques. In Test 1, 85\% of gage events were located by AIC with $\mathrm{a} \pm 4 \mathrm{~mm}$ deviation from manual determination whereas in Test 2 , only $70 \%$ of gage events were located with the same accuracy.

The performance of both signal onset determination techniques was also evaluated in terms of accuracy in gage events selection. As mentioned earlier, when only the top and bottom sensors are used, gage events can be selected based on their location along the longitudinal axis (the gage section corresponding to $\pm 12.7 \mathrm{~mm}$ from the center). The selected events were compared to those triggering the middle sensor first (Table 1). True gage events correspond to events with location within $12.7 \mathrm{~mm}$ from center that indeed triggered the middle sensor first whereas false gage events have a location wrongfully determined within $12.7 \mathrm{~mm}$ from center but did not trigger the middle sensor first. In Test 1, locationbased selection gives very accurate results, more than $90 \%$ of the actual gage events being selected. The AIC outperforms the first-threshold technique leading to the selection of $3 \%$ more true gage events and $3 \%$ less false gage events. In Test 2, the difference between first-threshold and AIC is more significant. 
Table 1. Selection of gage events based on location for two tensile tests on $\mathrm{SiC} / \mathrm{SiC}$ composites. For each are given the number of events and percentage of total number of gage events. True gage events correspond to events located within the gage section that indeed triggered the middle sensor first. False gage events correspond to events located within the gage section that did not actually trigger the middle sensor first.

\begin{tabular}{|c|c|c|}
\hline & Test 1 & Test 2 \\
\hline Manual & \multicolumn{2}{|l|}{} \\
\hline Gage events & 1050 & 1270 \\
\hline AIC & \multicolumn{2}{|l|}{} \\
\hline True gage events & $981(93 \%)$ & $950(75 \%)$ \\
\hline False gage events & $37(3 \%)$ & $79(6 \%)$ \\
\hline First threshold & \multicolumn{2}{|l}{} \\
\hline True gage events & $949(90 \%)$ & $848(67 \%)$ \\
\hline False gage events & $63(6 \%)$ & $317(25 \%)$ \\
\hline
\end{tabular}

Decrease in coupling quality leads to the selection of a lower fraction of true gage events. With AIC, $75 \%$ of the true gage events are selected against $67 \%$ with first-threshold. The main difference appears on the false gage events, which are wrongfully located within the gage section. While the AIC maintains a low number of false gage events (6\% of the total number of gage events), the first-threshold technique leads to the selection of $25 \%$ of false gage events.

To conclude the comparison between first-threshold and AIC-based techniques, performance evaluation based on location accuracy shows similar results because it uses gage events for which the waveforms are well defined and signal onset is clear and therefore it can be determined rather accurately even with the first-threshold technique. However, in terms of performance in gage events selection, AIC clearly outperforms the first-threshold technique especially when sensor coupling quality is not optimum, reducing the loss of information as well as the addition in the analysis of events originating from outside the gage section.

\subsection{Energy-based selection of AE events generated by damage}

The contribution of the energy-based approach to the selection of events is discussed for Test 1. First, the energy ratio vs. location plot was generated with manually-determined locations and a clear linear trend can be observed (Fig. 8a). However, the scatter is significantly greater than that observed on $\mathrm{C}_{\mathrm{f}} / \mathrm{C}-\mathrm{SiC}$ composites. The reasons for this are twofold. First, the frequency centroid of signals recorded after limited propagation throughout the material $(0$ to $20 \mathrm{~mm})$ ranged from 600 to $1200 \mathrm{kHz}$, which is almost twice the frequency range observed on $\mathrm{C}_{\mathrm{f}} / \mathrm{C}$-SiC composites, most likely due to the generation of $\mathrm{AE}$ events from various damage modes. Second, the matrix experiences significant damage during the tensile test in the form of transverse matrix cracks, which are expected to cause an increase in energy attenuation due to increased wave scattering. Both effects are illustrated in Fig. 8b, which represents the evolution of attenuation coefficient throughout test as a function of applied stress. Attenuation coefficient values were extracted from energy ratio vs. location plots generated for 250 consecutive gage events, every 100 events, considering for each waveform either the raw signal energy or the energy contained in a limited frequency interval $(50-500 \mathrm{kHz}$ or $1500-2000 \mathrm{kHz})$. At any given stress, attenuation is significantly greater at higher frequency, similar to what was observed on $\mathrm{C}_{\mathrm{f}} / \mathrm{C}$-SiC composites although attenuation coefficient values are much lower in $\mathrm{SiC} / \mathrm{SiC}$ composites overall. In addition, a significant increase in attenuation is observed throughout test, which is in good agreement with results reported in the literature for $\mathrm{SiC}_{\mathrm{f}} /[\mathrm{Si}-\mathrm{B}-\mathrm{C}]$ composites [4]. The present results highlight the need to account for frequency and damage dependence of attenuation when attempting to identify damage modes using AE. They should now be validated by comparison to ultrasonic measurements performed throughout tests. 


\section{MATEC Web of Conferences}

(a)
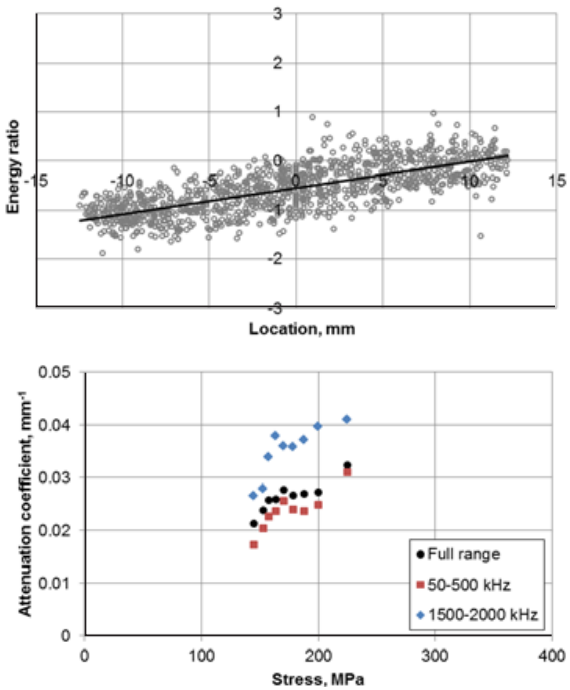

(b)

Figure 8. (a) Energy ratio vs. event location along the longitudinal axis (calculated from manually-determined signal onsets) and (b) evolution of attenuation coefficient throughout test for various frequency ranges, obtained from $\mathrm{AE}$ events generated during room temperature tensile test of a SiC/SiC composite.

(a)
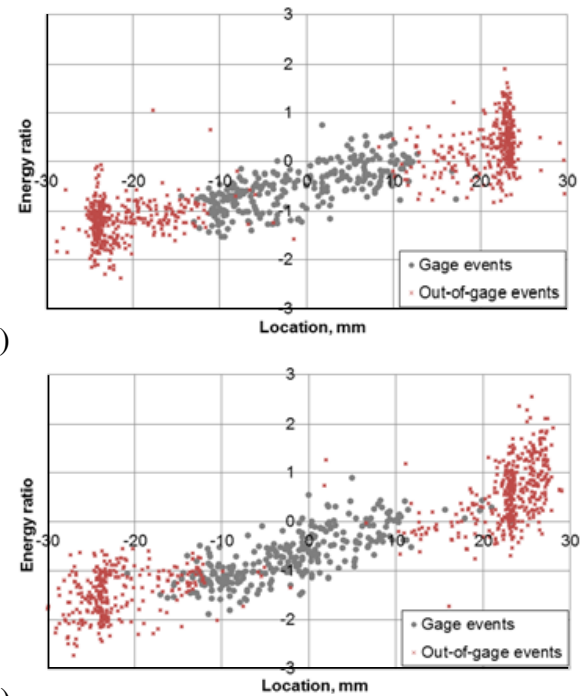

(b)

Figure 9. Energy ratio vs. event location for (a) the first 250 gage events and (b) the last 250 gage events generated during room temperature tensile test of a $\mathrm{SiC} / \mathrm{SiC}$ composite. Locations were determined automatically using AIC.

At last, energy ratio vs. location plots were generated automatically using the AIC technique for location determination. Figure 9 shows two plots corresponding to the first and last 250 gage events, respectively.

As mentioned earlier, the AIC technique provides very accurate selection of gage events. In Test 1 , only about 40 events are wrongfully located within the gage section. An automated selection of gage 
events could be set using for instance a confidence interval around the linear regression. Only those events within the confidence interval would be considered as gage events. The events outside of the admissible interval could either be discarded or manually inspected. In the present case, manual inspection is acceptable since only about 20 events appear clearly away from the linear trend within the gage section throughout the entire test. These events may correspond to noise signals or damage related events for which automatic onset determination failed to find the actual signal onset. Their selection or location could then be manually adjusted.

\section{Conclusion}

In the present paper, a waveform-based analysis of acoustic emission data that combines accurate event location and comparison of recorded energies was employed in order to select AE events originating from damage and characterize effects of propagation on the recorded waveforms. The energy-based approach validates that $\mathrm{AE}$ events originated from damage, therefore providing robust data for subsequent analysis such as damage mode identification. The approach only requires two remote sensors and is thus applicable when specimen geometry or testing conditions prevent placing a sensor in the middle of the gage section. It was demonstrated using artificial sources and then used on two different composite systems. The second benefit of the energy-based approach is the ability to characterize insitu energy attenuation and its dependence on frequency and damage progression using only AE events recorded during mechanical tests. Results obtained in $\mathrm{C}_{\mathrm{f}} / \mathrm{C}$-SiC composites are in excellent agreement with ultrasonic measurements. The energy-based approach now allows for a more accurate description of AE sources properties taking into account effects of propagation. Finally, the AIC-based AE event location determination was shown to outperform the so-called first-threshold-crossing technique, being less dependent on the quality of material/sensor coupling. Up to $85 \%$ of events were located with a deviation from manual determination not exceeding $4 \mathrm{~mm}$. Moreover, using the AIC-based technique to select events originating from the gage section ensured accurate selection, maximizing the number of selected true gage events and limiting the addition of false gage events to the analysis.

The authors gratefully acknowledge Dr. Mrityunjay Singh from the Ohio Aerospace Institute for providing the $\mathrm{C}_{\mathrm{f}} / \mathrm{C}$ $\mathrm{SiC}$ specimens, Rolls-Royce Corporation (Indianapolis, IN) for providing the $\mathrm{SiC} / \mathrm{SiC}$ specimens and Christopher Baker for helping with mechanical testing.

\section{References}

[1] G.N. Morscher, Compos. Sci. Technol. 59 (1999)

[2] G.N. Morscher, Compos. Sci. Technol. 64 (2004)

[3] E. Maillet, N. Godin, M. R’Mili, P. Reynaud, J. Lamon, G. Fantozzi, Comp. Sci. Technol. 72 (2012)

[4] E. Maillet, N. Godin, M. R’Mili, P. Reynaud, G. Fantozzi, J. Lamon, J. Eur. Ceram. Soc. 34 (2014)

[5] R. Gutkin, C.J. Green, S. Vangrattanachai, S.T. Pinho, P. Robinson, P.T. Curtis, Mech. Syst. Signal Pr. 25 (2011)

[6] M.G.R. Sause, A. Gribov, A.R Unwin, S. Horn, Pattern Recogn. Lett. 33 (2012)

[7] M. Moevus, N. Godin, M. R’Mili, D. Rouby, P. Reynaud, G. Fantozzi, G. Farizy, Comp. Sci. Technol. 68 (2008)

[8] E. Maillet, N. Godin, M. R’Mili, P. Reynaud, G. Fantozzi, J. Lamon, Compos. Part A 57 (2014)

[9] E. Maillet, G.N. Morscher, Mech. Syst. Signal Pr. 52 (2015)

[10] G.N. Morscher, Rev. Prog. QNDE 19 (2000)

[11] M.R. Gorman, Impact response and elastodynamics of composites (1990) 


\section{MATEC Web of Conferences}

[12] P. Sedlak, Y. Hirose, M. Enoki, Mech. Syst. Signal Pr. 36 (2013)

[13] T.D. Lhermitte, S.M. Handley, M.R. Holland, J.G. Miller, Ultrason. (1991)

[14] W. Huang, S.I. Rokhlin, Y.J. Wang, Ultrasonics 33 (1995)

[15] S. Biwa, Y. Watanabe, N. Ohno, Comp. Sci. Technol. 63 (2003)

[16] G.N. Morscher, A.L. Gyekenyesi, Comp. Sci. Technol. 62 (2002) 